Pacific Journal of Mathematics

F ON THE OPEN MAPPiNG THE OREN 


\title{
NOTE ON THE OPEN MAPPING THEOREM
}

\author{
LAWRENCE G. BROWN
}

\begin{abstract}
The open mapping and closed graph theorems are usually stated in terms of metrizable topological groups which are complete in a one-sided uniformity. It would be desirable to use only the weaker hypothesis of completeness in the two-sided uniformity. Important results of this sort are already known, and it is our purpose to strengthen those results by removing the separability hypotheses.
\end{abstract}

There are three uniformities normally used in connection with a topological group: the left, right, and two-sided uniformities. Completeness in the left uniformity is equivalent to completeness in the right uniformity, and stronger than completeness in the two-sided uniformity. If the group is metrizable, then it always has at least one left invariant metric; completeness in the left uniformity is equivalent to completeness in this metric (it does not matter which left-invariant metric is chosen). Still in the case of a metrizable group, completeness in the two-sided uniformity is equivalent to topological completeness, i.e., the existence of at least one metric in which the group is complete as a metric space (see [3], Exercise $Q(d)$, p. 212 for proof). Every topological group has a completion in the two-sided sense, but not necessarily in the one-sided sense (more precisely, the completion in the left-uniformity, though it is a complete uniform space, is not in general a group). When the one-sided completion does exist, it coincides with the two-sided completion. Two important examples of metrizable topological groups which are complete in the two-sided but not the one-sided sense are the full permutation group of a countably infinite set and the unitary group of an infinite-dimensional, separable Hilbert space (with the strong operator topology). Both of these examples are separable. Nonseparable examples of less naturality can be obtained by taking the direct product of one of the above with a nonseparable Banach space.

Lemma 1 below, which is the separable case of our theorem, was essentially proved by Banach [1], though his statement of it (Satz 8) was weaker. Its present statement and brief proof are the same as in Corollary 3.2 of Pettis [5]. Corollary 1 of the theorem and the corollary to Lemma 1 have some independent interest. They state that if $N$ is a closed normal subgroup of the group $G$, which is metrizable and complete in its two-sided uniformity, then $G / N$ is also complete. The corresponding result for one-sided completeness is trivial and is true even if $N$ is not normal. (This case where $N$ is 
not normal is, so far as we know, the only case where the results for one-sided completeness remain significantly superior to those for two-sided completeness.) Lemma 2, which is corollary 2 to the theorem, may also have some independent interest. Our theorem itself, when strengthened by the addition of the accompanying remark, differs from the "standard" version of the open mapping theorem ([2], Theorem 3, p. 90) only in the type of completeness used. Our version of the closed graph theorem, Corollary 3, is slightly weaker than the analog of the "standard" version in that both groups are assumed metrizable.

LEMma 1. Let $G$ and $H$ be separable metrizable topological groups which are complete in their two-sided uniformities and $\pi$ : $G \rightarrow H$ a continuous homomorphism such that $\overline{\pi(U)}$ is a neighborhood of the identity for each neighborhood $U$ of the identity in $G$. Then $\pi$ is open.

REMARK. It is easy to see that the hypothesis on $\overline{\pi(U)}$ will be satisfied if $\pi$ is surjective. This special case is Satz 8 of [1].

Proof. Let $U$ be an open nighborhood of the identity in $G$. Then $\pi(U)$ is analytic and hence ([4], p. 94-95) almost open. But $\pi(U)$ is second category in $H$. (If $\pi(U) \subseteq \bigcup_{n} F_{n}, F_{n}$ closed, then some $\pi^{-1}\left(F_{n}\right) \cap U$ has interior. Hence $\overline{\pi\left(\pi^{-1}\left(F_{n}\right)\right.} \subseteq F_{n}$ has interior.) Hence (see [5] or [3], Exercise $P(b)$, p. 211) $\pi(U) \cdot \pi(U)^{-1}$ is a neighborhood of the identity in $H$.

Corollary. If $G$ is a polonais group (i.e., a separable, metrizable, and topologically complete topological group) and $N$ is a closed normal subgroup, then $G / N$ is again complete and hence polonais.

Proof. Let $H$ be the completion of $G / N$.

Lemma 2. Let $G$ and $H$ be metrizable topological groups which are complete in their two-sided uniformities and $\pi: G \rightarrow H$ a continuous homomorphism such that $\overline{\pi(U)}$ is a neighborhood of the identity in $H$ for each neighborhood $U$ of the identity in $G$ and such that $\overline{\pi(G)}=$ $H$. Then for each closed separable subgroup $H_{0}$ of $H$, there is a closed separable subgroup $G_{0}$ of $G$ such that $\pi$ is a relatively open map of $G_{0}$ onto $H_{0}$.

\footnotetext{
1 A perhaps better choice for the "standard" version appears in [3; p. 213]. It has a weaker hypothesis.
} 
Proof. Let $U_{1} \supseteq U_{2} \supseteqq U_{3} \cdots$ be a fundamental system of neighborhoods of the identity in $G$. Let $V_{j}(j=1,2, \cdots)$ be open neighborhoods of the identity in $H$ such that $\overline{\pi\left(U_{j}\right)} \supseteqq V_{j}$. Let $G_{1}$ be a closed separable subgroup of $G$ such that $\overline{\pi\left(G_{1}\right)} \supseteqq H_{0}$ and

$$
\overline{\pi\left(G_{1} \cap U_{j}\right)} \supseteqq H_{0} \cap V_{j} \text {. }
$$

Let $H_{1}=\overline{\pi\left(G_{1}\right)}$. Similarly, define $G_{n}(n=2,3 \cdots)$ and $H_{n}(n=2,3 \cdots)$ so that for $n \geqq 1$ :

(i) $G_{n} \sqsubseteq G_{n+1}, H_{n} \sqsubseteq H_{n+1}$,

(ii) $\overline{\pi\left(G_{n}\right)}=H_{n}$,

(iii) $\overline{\pi\left(G_{n} \cup U_{j}\right)} \supseteqq H_{n-1} \cap V_{j}$,

(iv) $G_{n}, H_{n}$ are closed and separable.

Let $G_{\infty}=\overline{\mathrm{U}_{n=1}^{\infty} G_{n}}$ and $H_{\infty}=\overline{\mathrm{U}_{n=1}^{\infty} H_{n}}$. Then by Lemma $1, \pi$ is a relatively open map of $G_{\infty}$ onto $H_{\infty}$. Let $G_{0}=\pi^{-1}\left(H_{0}\right) \cap G_{\infty}$.

THEOREM. Let $G$ and $H$ be metrizable topological groups which are complete in their two-sided uniformities and $\pi: G \rightarrow H$ a continuous homomorphism such that $\overline{\pi(U)}$ is a neighborhood of the identity in $H$ for each neighborhood $U$ of the identity in $G$. Then $\pi$ is open.

REMARK. It is easy to remove all hypotheses on $H$ (except that $H$ be a Hausdorff topological group) from the statement of the theorem. (It is easy to construct a countable base at $e$ and hence prove $H$ metrizable. $H$ can then be completed.)

Prooj. Since $\overline{\pi(G)}$ is an open subgroup of $H$, we may assume $\pi(G)$ dence. Let $y_{n}$ be a sequence in $H$ so that $\lim y_{n}=e$. Then $\left\{y_{n}\right\}$ is imbedded in a closed separable subgroup $H_{0}$ of $H$. Choose $G_{0}$ as in Lemma 2. Then there are $x_{n}$ in $G_{0}$ such that $\pi\left(x_{n}\right)=y_{n}$ and $\lim x_{n}=e$.

COROLlary 1. If $G$ is a metrizable topological group which is complete in its two-sided uniformity and $N$ a closed normal subgroup, then $G / N$ is complete in its two-sided uniformity.

Corollary 2. With $G, N$ as in Corollary 1, any closed separable subgroup of $G / N$ is the image of a closed separable subgroup of $G$.

CoRollary 3. If $G$ and $H$ are as in the thorem, and $f: G \rightarrow H$ is a homomorphism such that $f$ has a closed graph, and $f^{-1}(U)$ is a neighborhood of the identity in $G$ for each neighborhood of the identity $U$ in $H$, then $f$ is continuous. 
Proof. Apply the theorem to the projection of $K$ onto $G$, where $K$ is the graph of $f$.

\section{REFERENCES}

1. S. Banach, Metrische Gruppen, Studia Math., 3 (1931), 101-113.

2. T. Husain, Introduction to Topological Groups, W. B. Saunders, Philadelphia, 1966.

3. J. Kelley, General Topology, Van Nostrand, Princeton, 1955.

4. K. Kuratowski, Topology Vol. I (translated from the French by J. Jawarowki), Academic Press, New York, 1966.

5. B. Pettis, On continuity and openness of homomorphisms in topological groups, Ann. of Math., 54 (1950), 293-308.

Received February 4, 1970.

STANFORD UNIVERSITY 


\section{PACIFIC JOURNAL OF MATHEMATICS}

\section{EDITORS}

H. SAMELSON

Stanford University

Stanford, California 94305

C. R. Hовву

University of Washington

Seattle, Washington 98105
J. DUGUNDJI

Department of Mathematics

University of Southern California

Los Angeles, California 90007

RICHARD ARENS

University of California

Los Angeles, California 90024

\section{ASSOCIATE EDITORS}
E. F. BECKENBACH
B. H. NeumanN
F. WOLF
K. YoSHIDA

\section{SUPPORTING INSTITUTIONS}

UNIVERSITY OF BRITISH COLUMBIA

CALIFORNIA INSTITUTE OF TECHNOLOGY

UNIVERSITY OF CALIFORNIA

MONTANA STATE UNIVERSITY

UNIVERSITY OF NEVADA

NEW MEXICO STATE UNIVERSITY

OREGON STATE UNIVERSITY

UNIVERSITY OF OREGON

OSAKA UNIVERSITY

UNIVERSITY OF SOUTHERN CALIFORNIA
STANFORD UNIVERSITY

UNIVERSITY OF TOKYO

UNIVERSITY OF UTAH

WASHINGTON STATE UNIVERSITY

UNIVERSITY OF WASHINGTON

AMERICAN MATHEMATICAL SOCIETY CHEVRON RESEARCH CORPORATION NAVAL WEAPONS CENTER 


\section{Pacific Journal of Mathematics}

\section{Vol. 38, No. $1 \quad$ March, 1971}

Bruce Alan Barnes, Banach algebras which are ideals in a Banach algebra ..... 1

David W. Boyd, Inequalities for positive integral operators............... 9

Lawrence Gerald Brown, Note on the open mapping theorem .............. 25

Stephen Daniel Comer, Representations by algebras of sections over Boolean

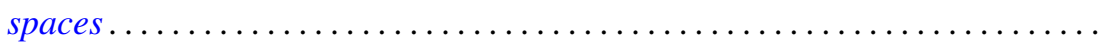

John R. Edwards and Stanley G. Wayment, On the nonequivalence of

conservative Hausdorff methods and Hausdorff moment sequences ........

P. D. T. A. Elliott, On the limiting distribution of additive functions $(\bmod 1) \ldots \ldots$

Mary Rodriguez Embry, Classifying special operators by means of subsets

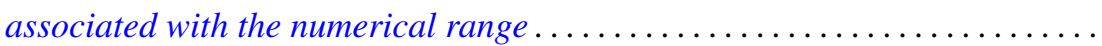

Darald Joe Hartfiel, Counterexamples to a conjecture of G. N. de Oliveira ......

C. Ward Henson, A family of countable homogeneous graphs...............

Satoru Igari and Shigehiko Kuratsubo, A sufficient condition for

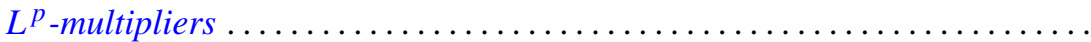

William A. Kirk, Fixed point theorems for nonlinear nonexpansive and

generalized contraction mappings............................

Erwin Kleinfeld, A generalization of commutative and associative rings ...... 95

D. B. Lahiri, Some restricted partition functions. Congruences modulo $11 \ldots \ldots 103$

T. Y. Lin, Homological algebra of stable homotopy ring $\pi *$ of spheres ....... 117

Morris Marden, A representation for the logarithmic derivative of a meromorphic function...........................

John Charles Nichols and James C. Smith, Examples concerning sum properties for metric-dependent dimension functions . .

Asit Baran Raha, On completely Hausdorff-completion of a completely

Hausdorff space.

M. Rajagopalan and Bertram Manuel Schreiber, Ergodic automorphisms and affine transformations of locally compact groups..........

N. V. Rao and Ashoke Kumar Roy, Linear isometries of some function

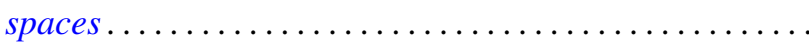

William Francis Reynolds, Blocks and F-class algebras of finite groups

Richard Rochberg, Which linear maps of the disk algebra are multiplicative ...

Gary Sampson, Sharp estimates of convolution transforms in terms of decreasing

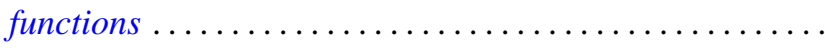

Stephen Scheinberg, Fatou's lemma in normed linear spaces

Ken Shaw, Whittaker constants for entire functions of several complex

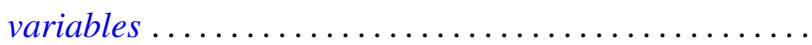

James DeWitt Stein, Two uniform boundedness theorems................ 251

$\mathrm{Li} \mathrm{Pi} \mathrm{Su,} \mathrm{Homomorphisms} \mathrm{of} \mathrm{near-rings} \mathrm{of} \mathrm{continuous} \mathrm{functions} \mathrm{.} \mathrm{.............} 261$

Stephen Willard, Functionally compact spaces, $C$-compact spaces and mappings of minimal Hausdorff spaces....................... 